\title{
LA EVOLUCION PALEOGEOGRAFICA DE LOS LLANOS LERIDANOS (SECTOR ORIENTAL DE LA DEPRESION DEL EBRO) DURANTE EL CUATERNARIO
}

\author{
José Luis PEÑA MONNE \\ Universidad de Zaragoza
}

\begin{abstract}
RESUMEN: En el sector oriental de la Depresión del Ebro se ha individualizado una gran depresión erosiva, caraclerizada por la planitud general (Plá de Lleida, Plá d'Urgell) en donde se reconoce un sistema escalonado de 9 terrazas cuaternarias, como consecuencia de la alternancia de fases dinámicas contrastadas, asi como cambios importantes en su dispositivo paleogeografico. especialmente en el Plá d'Urgell y en el Noguera Ribagorzana.
\end{abstract}

\begin{abstract}
A major erosive depression characterized by its general planitude (Pla de Lleida, Plá d'Urgell) has been individualized in the eastern sector of the Ebro Basin. There, as a consequence of pronounced alternating dynamic phases, a system of nine quaternary terrace fligths may be recognized, as well as important changes in the palaeogeographic pattern of the Pla dUrgell and Noguera-Ribagorzana areas.
\end{abstract}

Sumario: Introducción.- La evolución precuatcrnaria.- La evolución cuaternaria.- La evolución palcogeográfica.- Bibliografía.

\section{INTRODUCCION}

Los Llanos leridanos abarcan las comarcas de menor altitud del sector oriental de la Depresión del Ebro, cerrados al Norte por las Sierras Exteriores Pirenaicas y al Sur y Este por los relieves monoclinales de La Segarra y Les Garrigues, que forman el tránsito topográfico hacia la Cordillera Costera Catalana. Su rasgo más definitorio es la planitud general (Plá de Lleida, Urgell), que se prolonga igualmente hacia el valle del Cinca (La Llitera), y que viene determinada por la conservación de extensos mantos acumulativos depositados a lo largo del Cuaternario por los ríos procedentes de las áreas montañosas marginales de esta cuenca erosiva, de la que el rio Segre constituye el eje directriz. La gran dispersión espacial de los depósitos cuaternarios en un área tan extensa y las diferentes procedencias de los materiales dificulta extraordinariamente su correlación gencral. Sin cmbargo, sólo a partir de la cartografía geomorfológica realizada con un carácıer exıensivo en los últimos años (PEÑA, 1988; SANCHO, 1988; PEÑA y SANCHO, 1988) podemos aproximarnos a un conocimiento mejor de la evolución y los cambios palcogeográficos producidos en esta región a lo largo del Cuatcrnario. 


\section{LA EVOLUCION PRECUATERNARIA}

La evolución geológica de este sector oriental de la Depresión del Ebro comienza a partir de la individualización de la cuenca en el Priaboniense superior, en que los sedimentos dejan de ser de origen marino para convertirse durante todo el Oligoceno y Neogeno en depósitos continentales. Los materiales aflorantes más antiguos en este área pertenecen al Eoceno terminal y constituyen la $F m$. Yesos de Barbastro, que afloran diapíricamente en los ejes de los anticlinales que limitan por el Norte y NE estos Llanos: anticlinal de Alfarrás-Balaguer (Serra Llarga) y de la Serra d Almenara. Por encima de esta formación se encuentran series molásicas y calcáreas que atestiguan una situación evolutiva continental con una actividad erosivoacumulativa fluvial. En los márgenes de la cuenca se depositaron los conglomerados de Berga y Montsant y del Alt Rialb ( $F m$. Molasas de Solsona), que pasan a facies intermedias y distales hacia el área de estudio, correspondientes a depósitos finos de llanura de inundación sobre los que se han jerarquizado algunos surcos, posteriormente rellenos en forma de paleocanales arcnosos, con fuerte migración lateral. Estas facies constituyen la Formación Urgell, corrclativa de la Fm. Peraltilla del sector aragonés. Las molasas distales de la Fm. Urgell pasan lateralmente hacia ambientes sedimentarios de carácter lacustre-salobre, con facies carbonatadas que configuran la $F m$. Calizas de Tárrega (RIBA,1967) y a los lignitos y calizas del sector meridional (Fm. Calizas de Castelldans, Fm. Calizas de Almatret y de Mequinenza, según RIBA, 1972 ). La edad de estos sedimentos es claramente oligocena (RIBA et al, 1983) estando ausentes de esta zona los materiales neógenos, que sin embargo afloran más al Oeste (rio Cinca).

La disposición estructural de estos sedimentos detríticos y carbonatados continentales es prácticamente horizontal, aunque en conjunto existe un claro buzamiento de las capas hacia el Ocstc. Las mayores deformaciones se encuentran en las proximidades del núcleo de yesos del anticlinal septentrional y nordoriental (Alfarrás- Balaguer-Artesa de Segre y La SentiuAlmcnara), con capas subverticales, aunque los materiales recuperan la subhorizontalidad en un corto tramo. También adquicren cierta importancia en los márgenes de La Segarra, donde conforman relieves monoclinales de calizas, con suaves buzamientos hacia el NW. Tales deformaciones son debidas a las fases tectónicas post-Oligocenas.

\section{LA EVOLUCION CUATERNARIA}

Frente al relleno continuado de la Depresión del Ebro generado con anterioridad al Mioceno, la evolución posterior se ha caracterizado por un constante desalojo de matcriales, debido a la cxcavación fluvial. Esta dinámica se ha prolongado igualmente a lo largo del Cuatternario, aprovechando los matcriales más blandos de la depresión terciaria, dando lugar a la configuración de una cuenca crosiva (Plá de Llcida y d'Urgeli), cuyo fondo se encucntra entre los 100 y $200 \mathrm{~m}$. de altura y donde escasean los relieves residuales de carácter estructural y abundan las acumulaciones cuaternarias. La cucnca está rodeada de relicves más vigorosos modelados en los matcriales detríticos y carbonatados oligocenos, de mayor resistencia, que conforman alineaciones estructurales al Sur de La Noguera, en La Segarra y Les Garrigues, así como por las sicrras modeladas en los anticlinales del sector Norte y Nordeste (Serra Llarga y (de Almenara), que se colocan por encima de $\operatorname{los} 450 \mathrm{~m}$. de altitud y se prolongan por las Sicras Exicriores Pircnaicas. 
La red fluvial dirigida por los rios Segre y Cinca debió instalarse ya en el Plioceno (PEÑA, 1983), en un proceso de sobreexcavación que vació parcialmente este sector de la cuenca. Desde el punto de vista hidrográfico, constituye una importante zona de confluencia fluvial, formada por el rio Segre y sus afluentes principales en este área: en la orilla derecha, el Farfanya y el Noguera Ribagorzana; en el margen izquierdo, el Sió, Ondara, Corb, Femosa y Set. Poco más al SW, el Segre se une con el Cinca y más allá con el rio Ebro, que constituye el nivel de base general de la Depresión.

La morfología de detalle nos muestra la destacada importancia que poseen los materiales cuaternarios en el modelado, ya que conforman extensas plataformas, como las del sector occidental, formadas por las terrazas fluviales altas del Noguera Ribagorzana, o las plataformas de detritus calcáreos de los conos altos del Femosa y Ondara. Es muy interesante constatar la existencia de una continuada inversión del relieve, que ha permitido la conservación de terrazas fluviales, especialmente los niveles medios, bordeando los rios principales (Segre y Noguera Ribagorzana), formando una especie de muralla sobreelevada frente a la mayor excavación de los relieves margoarcillosos en los que se apoyaban estos cauces fluviales, que han sido desmantelados por completo, modelándose depresiones de contacto ocupadas por los niveles de conos y glacis más bajos, caso de la depresión entre las terrazas del Noguera y Segre (depresión del Farfanya) o entre los niveles culminantes y las terrazas medias del Noguera (depresión del Segriá) o las zonas bajas situadas trás las terrazas del Segre que constituyen la prolongación del Plá d'Urgell y del Plá de Lleida

El dispositivo paleogeográfico se mantiene con idénticas características a lo largo del Cuaternario, aunque a nivel de detalle la actividad fluvial se ve marcada por la alternancia de etapas acumulativas y de incisión, ligadas a las fluctuaciones climáticas pleistocenas, que han determinado la existencia de un escalonamiento generalizado de niveles de terraza en los rios principales y la presencia de varias generaciones de abanicos aluviales en los rios del Plá d'Urgell y en el rio Farfanya. Pero, además, desde el punto de vista evolutivo, se generaron cambios en la disposición de los cursos fluviales, especialmente en el rio Noguera Ribagorzana y en los rios Set, Femosa, Corb y Ondara.

\section{Las terrazas escalonadas de los rios Segre y Noguera Ribagorzana}

Entre Balaguer y la confluencia con el Cinca, el rio Segre amplia notablemente su valle tras salir de las Sierras Exteriores Pirenaicas a partir de Camarasa y Sant Llorenç. De idéntica forma lo hace el Noguera Ribagorzana a partir de la cluse de Ivars de Noguera y hasta su confluencia con el rio Segre, en Corbins. Es por ello la zona de mayor cantidad de acumulaciones cuaternarias de terrazas, aunque el número de niveles existentes se ha prestado a múltiples valoraciones (SOLE y FONT, 1929; SOLE, 1946, 1953 a,b; CALVET, 1980, 1986; PENA, 1983, 1988). Queda clara la presencia de 6 niveles de terraza en el rio Segre, aunque podrían ampliarse a 8 si tenemos en cuenta, como scñalan PEÑA y SANCHO (1988) que los retazos de la antigua confluencia Segre-Noguera Ribagorzana, en los Tossals de La Brisa (Aitona), se sitúan a 90-100 m. (T 7) y a 115-120 m. (T8) (Cuadro 1).

El rio Noguera Ribagorzana también presenta grandes confusiones en sus niveles, aunque la mayor parte de éstos parecen coincidir con los del Segre, especialmente en el sector de conflucncia actual, ya que en el Pleistoceno inferior presentaba un trayecto distinto. Este rio, en su cvolución cuaternaria se ha caracterizado por presentar una pendiente muy superior a la 
del rio Segre, lo que se manifiesta igualmente en la pendiente general de sus depósitos. Ello ha sido debido al propio recorrido que presentan ambos rios y, por otra parte, al proceso de captura producido en el Cuaternario antiguo, ya que las terrazas más altas (niveles 6-7, 8 y 9) se conservan en el interfluvio Segre-Cinca, habiendo sido incluso tributario de ese último río, tal como se desprende de su cartografía geomorfológica (BOMER, 1979; PEÑA, 1988).

La correlación de los sistemas de terraza fluvial del Segre y Noguera Ribagorzana con las del rio Cinca ha sido señalada por PEÑA y SANCHO (1988). Tal como se aprecia en el Cuadro 1, la correlación puede hacerse extensiva hasta el nivel 9 del Cinca y debe significar que la ausencia en el valle del Segre de los niveles más altos (T10 y T 11) es debida a la erosión. Toda la red Segre-Cinca ha tenido que evolucionar conjuntamente,por lo que el escalonamicnto que puede admitirse, como mínimo, es de 11 niveles de terraza.

\begin{tabular}{|c|c|c|c|}
\hline & CINCA & N.RIBAGORZ. & SEGRE \\
\hline T 1 & $2-3$ & 1'5- 8 & $1 \cdot 5-2$ \\
\hline T 2 & 10 & 10 & 10 \\
\hline T 3 & 20 & 20 & $18-20$ \\
\hline T 4 & 45 & $35-40$ & $35-40$ \\
\hline T 5 & 60 & $50-65$ & 60 \\
\hline T 6 & $85-90$ & \multirow{2}{*}{$90-100$} & $80-90$ \\
\hline$T 7$ & $100-105$ & & \\
\hline T 8 & $115-120$ & $115-120$ & \\
\hline T 9 & 150 & 150 & \\
\hline T 10 & 190 & & \\
\hline T 11 & 200 & & \\
\hline
\end{tabular}

Cuadro 1. Correjación altimćtrica entre las terrazas de los ríos Cinca, Noguera Ribagorzana y Segre (scgún PEÑA y SANCHO,1988)

Los depósitos cuaternarios de estos rios se caracicrizan por el dominio de los niveles de gravas poligénicas de procedencia pirenaica, abundando el granito, las rocas comcanas, pizarras, cuarcitas, calizas, areniscas. Aparecen intercalaciones de lentejones y niveles de arenas grises procedentes de la descomposición del granito y los limos ocres de inundación, 
tanto interestratificados como ocupando la parte superior de las acumulaciones de terraza. Las gravas son de tamaños entre 3 y $8 \mathrm{cms}$. predominantemente, con un elevado grado de rodamiento. Por otra parte, el sector de terrazas fluviales situado encima o en las inmediaciones del anticlinal de yesos de la Fm. Barbastro presenta deformaciones de carácter halocinético, señaladas por prácticamente todos los autores que han trabajado en esta zona, con diferentes valoraciones en su interpretación de detalle (véase entre otros a SOLE, $1953 \mathrm{a}, \mathrm{b}$; ALBERTO et al. 1983); la incidencia de esta acción pseudotectónica en la dinámica fluvial ha podido ser muy importante ${ }_{2}$ ya que parece ser la causa de los cambios de dirección del rio Segre en su curso medio (PENA, 1983).

\section{El cono del rio Farfanya.}

El rio Farfanya es un afluente del Segre que tiene su cuenca de recepción en las Sierras Marginales Pirenaicas. Sus acumulaciones más importantes se localizan en un amplio cono disimétrico desarrollado a la salida de la cluse de Castelló de Farfanya trás atravesar el anticlinal de yesos de la Serra Llarga. Este cono ocupa parte de una depresión erosiva excavada entre el frente de la Serra Llarga y los niveles de Terraza 5 del Noguera Ribagorzana y niveles 5 y 6 del Segre, en donde anteriormente existirían retazos de abanicos más antiguos que enlazarían con dichas terrazas. Aparte del fondo del valle actual, cuya llanura aluvial enlaza con el nivel T1b del Segre, el único resto acumulativo es el cono, que aunque no posee conexión topográfica directa, parece posible enlazarle con la terraza de $20 \mathrm{~m}$. del rio Segre. Aparte, encontramos restos de otro nivel de conos en los ápices colgados situados al pie de Serra Llarga, algo más al Este, cuya cronología deposicional podría tener relación con la Terraza 4 ó 5 del Segre.

\section{Los abanicos aluviales del Plá d’Urgell.}

Los afluentes de la orilla izquierda del Segre presentan características morfologicas y acumulativas totalmente distintas a las del piedemonte pirenaico. Son rios cortos, que circulan únicamente por el Terciario de la Depresión del Ebro y que por tanto presentan un espectro litológico en sus acumulaciones mucho mas restringido. Sus cabeceras están situadas en las calizas y margas de la Fm. Tárrega, modeladas en cuestas de escaso buzamiento, que configuran el paisaje característico de La Segarra. En el sector mas suroccidental, se trata de las plataformas de areniscas y calizas de los relives de Les Garrigues.

La red aparece ordenada en cinco colectores básicos: Sió, Ondara, Corb, Femosa y Set. Todos ellos discurren de Este a Oeste, desembocando en el rio Segre entre Balaguer y Sudanell. Al Sur de esta localidad se trata de barrancos de menor entidad y sin apenas acumulaciones de importancia. De estos ríos, el Sió es el único que presenta una morfología verdaderamente fluvial, sin abanicos aluviales terminales, ya que su valle de fondo plano queda cerrado marginalmente por alineaciones de relieves del anticlinal de Cubells al Norte, y de Almenara al Sur. Presenta tres niveles generales de terraza, con alturas de 1-2, 25 y $40 \mathrm{~m}$., aunque hay algunos restos más elevados, enlazando perfectamente con las terrazas del Segre, al Este de Balaguer. Los demás rios abandonan, en sus niveles altos, el área madre monoclinal entre 15 y $30 \mathrm{~km}$. antes de alcanzar la confluencia, dando origen a extensísimos abanicos, que a lo largo 
de su evolución cuaternaria han cambiado continuamente de posición, entrelazándose entre si para formar llanuras continuas, con pendientes del 4-4,5/mil, de tal manera que resulta difícil establecer de forma exacta los límites de cada uno de ellos.

Dentro de esta vasta extensión de conos de deyección podemos diferenciar numerosos niveles, siendo los más elevados los más degradados por la erosión y por tanto los de más compleja correlación. A ello hay que añadir el alejamiento que presentan estos niveles altos respecto de las terrazas del Segre, que obligan a establecer prolongaciones hipotéticas de sus respectivas pendientes. Desde los años 70 se han relacionado de forma clara estas acumulaciones con las terrazas del Segre (BOMER, 1978; CALVET, 1980, 1986; PEÑA, 1988).

La generalidad más importante de estas acumulaciones proviene de su composición litológica y sedimentológica, resultante de poseer una área madre común y de gran homogeneidad: las calizas de la Fm. Tárrega. Son depósitos de cantos calcáreos, con tamaños en tomo a 4-6 cms, forma aplanada y aristas algo redondeadas. La estratificación general es muy neta con niveles de cantos entre capas de arenas finas y lentejones arenoso-limosos. A techo abundan las costras calcáreas y los encostramientos por carbonatos, que endurecen la acumulación y han permitido su conservación en forma de plataformas y cerros aislados (GUTIERREZ et al., 1985). Creemos, como CALVET y GALLART (1979), que estas calizas angulosas no tienen necesariamiente un origen periglaciar, sino que la causa debe buscarse en el tableamiento y estructura de las calizas de Tárrega, fácilmente deleznables en clastos de tamaños pcqueños por la simple acción del arroyamiento, lo que no descarta que esa acción se viera todavía más acelerada en las fases frías cuaternarias por la intensidad de la gelifracción.

El problema inicial de correlación y definición de niveles lo plantea la gran dispersión que presentan los retazos de acumulaciones cuaternarias, ocupando diferentes sectores de tres hojas a cscala l: 50.000. Por su relación topográfica directa o indirecta con las terrazas del rio Segre se han diferenciado 6 niveles, teniendo caractcrísticas de conos en todos los rios, exceptuando los niveles más bajos del Femosa y Set, que adoptan una disposición en terrazas encajadas (Cuadro 2).

\begin{tabular}{|c|c|c|c|c|c|c|}
\hline & Nivel 6 & Nivel $5 / 5^{\prime}$ & Nivel 4 & Nivel 3 & Nivel 2 & Nivel 1 \\
\hline ONDARA & & & - & & & \\
\hline CORB & & - & - & & & \\
\hline FEMOSA & & & & & & \\
\hline SET & & & & & & \\
\hline
\end{tabular}

Cuadro 2. Niveles de conos (puntos densos) y de terraza (puntos amplios) de los rios del Plá d'Urgell. 


\section{Cronología de las acumulaciones cuaternarias.}

En los últimos años se han realizado hallazgos arqueológicos de una validez cronológica relativa en el rio Segre (SUNYER, 1973, 1974), en el valle del Femosa y Set (FULLOLA et al, 1985 a, b, 1986) y en el cono del Farfanya (MORA et al., 1986) que han permitido asentar algo más la posición cronológica (PEÑA, 1988) del sistema acumulativo pleistoceno (Cuadro 3), aunque la precariedad de los datos sólo permite conclusiones provisionales .

\begin{tabular}{|c|c|c|}
\hline Nivel & 6 & .................. Pre-Riss \\
\hline Nivel & 5 & ............... Riss \\
\hline Nivel & 4 & ................. Intergl. Riss-Würm \\
\hline Nivel & 3 & ................ Würm I-II \\
\hline Nivel & 2 & Würm IV \\
\hline Nivel & 1 & .................. Holoceno \\
\hline
\end{tabular}

Cuadro 3 . Cronología de los niveles de terraza del rio Segre (según PEÑA,1988).

\section{LA EVOLUCION PALEOGEOGRAFICA}

De la evolución precuatemaria y cuaternaria expuestas y de la disposición cartográfica de los niveles pleistocenos conservados, se pueden extraer los rasgos evolutivos esenciales de la paleogeografía del Cuaternario de los Llanos Leridanos. Como hemos indicado, desde el Plioceno existe una cubeta erosiva excavada en los materiales margo-arcillosos oligocenos, hacia la que drena la red del conjunto Cinca-Segre. Una sucesión de etapas de acumulación e incisión cuaternarias han ido construyendo y destruyendo sucesivamente el fondo de esta cuenca, al tiempo que se iban produciendo cambios notables en los dispositivos sedimentarios de esos rios. Por una parte, el rio Noguera Ribagorzana, durante la sedimentación de los niveles más altos ( 8 y 9) mostraba un continuo desplazamiento del cauce de Este a Oeste, materializándose en el nivel 6 (Fig. 1) con una difluencia hacia el Segre y Cinca simultáneamente, tal como lo demuestra el seguimiento de sus terrazas fluviales. Esta misma característica migratoria se observa en el rio Cinca desde sus terrazas más altas hasta las recientes (PEÑA y SANCHO, 1988). Repentinamente, antes del Riss, el Noguera abandonó el 
lecho del nivel 6 al ser capturado por un pequeño alluente del Segre, de manera que a el nivel 5 $c$ inferiores (todos ellos del Riss y posteriores) aparecen dispuestos con una nueva trayectoria que acortó su trazado, aunque sin variar apenas su pendiente, ya que pasó a desembocar a una cola mayor, dada su posición aguas arriba del Segre (Figs. 2 y 3 ).

Por otra partc, en los conos orientales, es constante a lo largo de la sedimentación pleistocena la migración lateral de los cauces, especialmente en los rios Set y Femosa, que finalmente pasan a convertirse en rios encauzados en sus últimas fases evolutivas (Figs. 2 y 3 ), con sedimentación de terrazas. GALLART et al (1984) han relacionado los cambios de dirección observados en los abanicos de otro de estos rios, el Corb, con basculamientos recientes hacia el NW de la parte Sur del Plá d’Urgell debidos a la tectónica distensiva neógena. De hecho, los deplazamientos obscrvados a lo largo del Plcistoceno en el Noguera y Cinca hacen sospechar una iniciativa tectónica, pero los datos existentes sobre la neotectónica cuaternaria en esta región son por ahora casi nulos.

Pese a estas variaciones laterales, propias de cursos de carácter torrencial, como son cualquiera de los rios que fluyen hacia estos llanos, podemos señalar la existencia general de 11 etapas acumulativas separadas por períodos de incisión. Aunque en el valle del Segre sólo se conservan 6 nivcles de terraza, el rio Nogucra proporciona testimonios de 9 niveles, que son completados por las 11 terrazas del rio Cinca. Dada la perfecta correlación mostrada por estas terrazas en los tres rios hasta el nivel 6, momento en el cual enlazaban directamente debido a la difluencia del Nogucra, podría tomarse estos 11 niveles como base de correlación general para cl Cuaternario del Plá de Lleida, Urgell, Litera y Bajo Cinca .

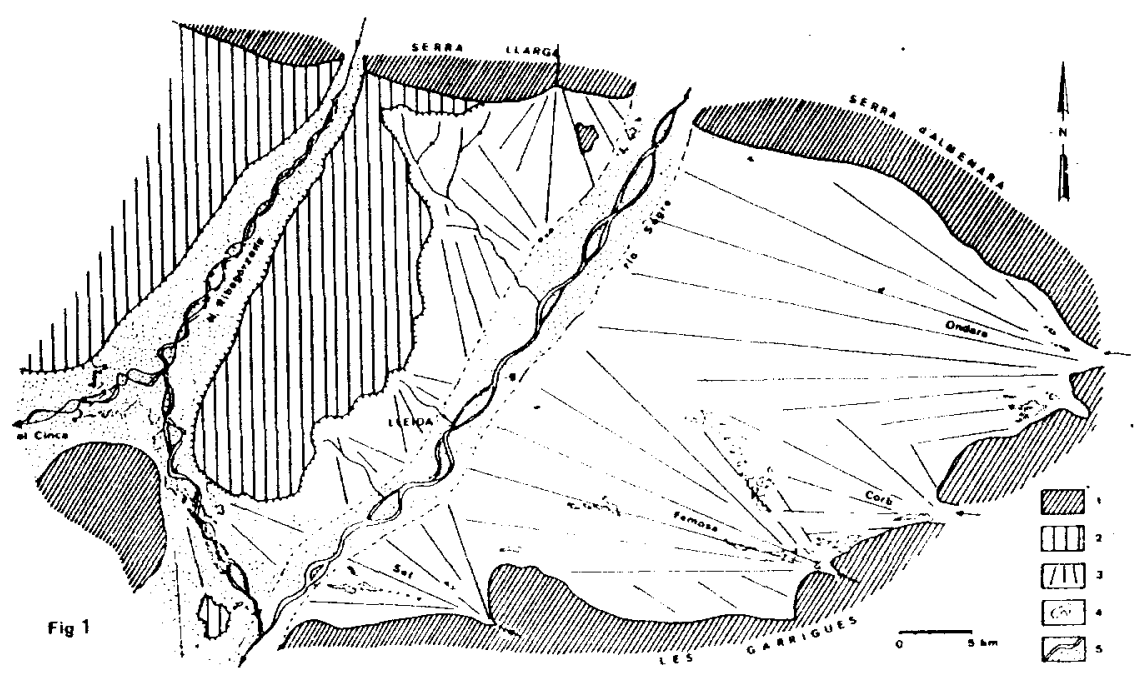

Fig.1. Reconstrucción paleogeográfica de los llanos leridanos durante la claboración del nivel de terrazas y conos 6. (1): Relieves estructurales marginales. (2): Niveles Cuaternarios anteriores al nivel 6. (3): Conos y glacis del nivel 6. (4): Retazos actuales del nivel 6. (5): Lechos fluviales del nivel 6. 


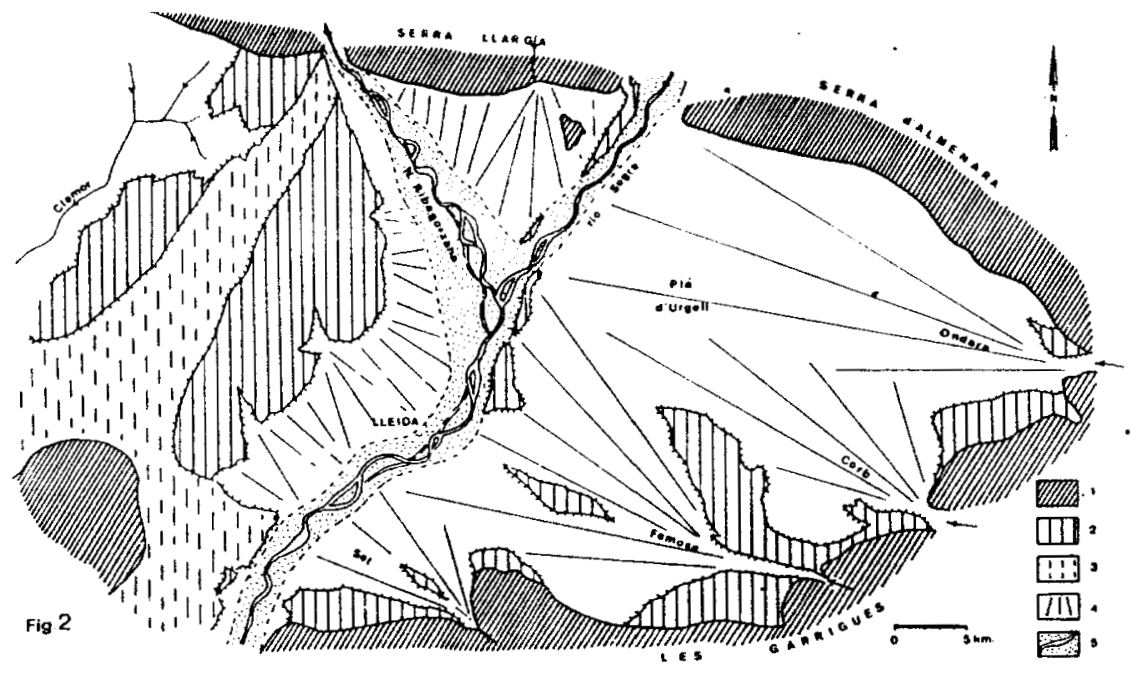

Fig. 2. Reconstrucción paleogeográfica de los llanos lcridanos durante la elaboración del nivel 5 . (1): Relieves estructurales marginales. (2): Niveles Cuaternarios anteriores al nivel 5. (3): Brazo abandonado del Noguera Ribagorzana. (4): Conos y glacis del nivel 5. (5): Lechos fluviales del nivel 5.

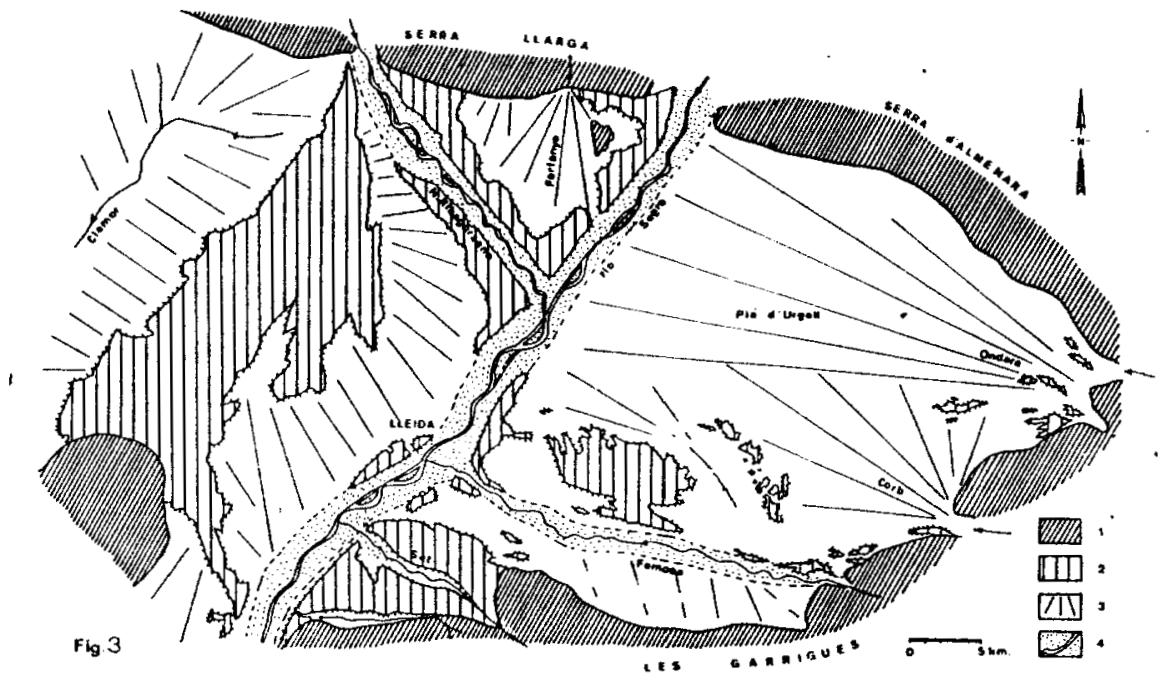

Fig.3. Reconstrucción paleogeográfica de los llanos leridanos durante la claboración del nivel 3. (1): relieves estructurales marginales. (2): Niveles Cuaternarios anteriores al nivel 3. (3): Conos y glacis del nivel 3. (4): Lechos fluviales del nivel 3. 


\section{BIBLIOGR AFIA}

ALbERTO, F., GUTIERREZ, M., IBAÑEZ, M.J., MACIIN, J., MELENDEZ, A., PEÑA, J.L., POCOVI, A. y RODRIGUEZ , J. (1983): "El piedemonte pliocuaternario en el sector central pirenaico (Huesca y Lérida)". Geographicalia , 18, pp.109-126.

BOMER, B. (1978): Le Bassin de l'Ebre et ses bordures montagneuses. Etude géomorphologique. Thèse Doctorat Univ. de Caen 3 t. 662 p. (Ined.).

BOMER, B. (1979): "Les piedmonts du Bassin de 1'Ebre (Espagne)". Méditerranée, 3, pp. 19-25.

CALVET, J. (1980): Relaciones entre la evolución geomorfológica cuaternaria de la Depresión Central Catalana (Segarra y Plá d'Urgell) y del valle del Segre. 102 p. Fundación J. March. Madrid. (Ined.).

CALVET, J. (1986): "Excursion to the Catalan Central Basin". I.G.U. Working Group on Geomorphological Survey and Mapping Meeting Spain. pp. 55-73.

CALVET, J. y GALLART, F. (1979): "Las brechas clacáreas del Plá d Urgell. Su repartición espacial e interpretación". Actas III Reunión Nac. G.E.T.C., pp. 117-121.

FULLOLA, J.M., GALLART, J., PEÑA, J.L. y GARCIA-ARGUELLES, P.(1985b): "El Paleolitic superior de la Bauma de la Peixera d'Alfés (Segriá, Lleida) (2* Campanya d"excavacions, octubre de 1984): noves perspectives a les comarques lleidatanes". T. I Hom. Dr. J.M. Corominas, pp. 169-179. Banyoles.

FUlLOLA, J.M., GALLART, J., PEÑA, J.L.y GARCIA-ARGUELlES, P. (1985 a): "El yacimiento paleolítico de la Bauma de la Peixera d'Alfés (Segriá, Lérida): noticia de la $1^{*}$ campaña de excavaciones (abril-1984)". Bajo Aragón, Prehistoria, V,pp. 159-168. Zaragoza.

FULLOLA, J.M., PENA, J.L., GALLART, J. y GARCIA-ARGUELLES, P.(1986): "La tercera campanya d'excavacions al jaciment del Paleolitic superior de la Bauma de la Peixera d'Alfés (Segriá), maig 1985 ". XVIII Jornades Grup Recerques Terres de Ponent, IX, pp.23-30.

GALLART, F., CALVET, J. y CLOTET, N. (1984): "Observaciones geomorfológicas en el sector sur del Plá d'Urgell, posible influencia de deformaciones tectónicas recientes en el origen de esta depresión". Acta Geol. Hisp. , 19 (2), pp. 75-80.

GUTIERREZ, M., MELENDEZ, A.y PEÑA, J.L. (1985): "Desarrollo de caliches en los glacis cuaternarios del Este de Lérida (sector oriental de la Depresión del Ebro)". I Reuniao do Quaternario Ibérico, t. II, pp . 287-300. Lisboa.

MORA, R., CARBONELL, E., MARTINEZ, J. y LLUSA, A.(1986): "Troballes acheulianes a la Conca del riu Farfanya". Ilerda, XLVIl, pp. 153-160. Lleida.

PEÑNA, J.L. (1983): La Conca de Tremp y Sierras Prepirenaicas comprendidas entre los rios Segre y Noguera Ribagorzana.Estudio geomorfológico. Inst. de Est. Ilerdenses. 373 p. Lérida.

PLÑA, J.L. (1988): Las acumulaciones cuaternarias de los Llanos leridanos. Aspectos generales e itinerarios de campo. Curso de Iniciación a la Geoarqueología. Institut d'Estudis Ilerdencs. 81 p. LLeida.

PIiÑA, J.L. y SANCHO, C. (1988): "Correlación y evolución cuatemaria del sistema fluvial Segre-Cinca en su curso bajo (provs. de Lérida y lluesca)". Cuaternario y Geomorfología, 2 (en prensa).

RIBA, O. (1967): "Resultados de un estudio sobre el Terciario continental de la parte Este de la depresión central catalana". Acta Geol.Hisp., 2 (1), pp. 3-8. Barcelona.

RIBA , O., REGUANT, S. y VILLLNA (1983) : "Ensayo de síntesis estratigráfica y evolutiva de la cuenca terciaria del Ebro". Libro Jubilar J.M. Rios, t. II, pp. 131-159. I.G.M.E. Madrid.

RIBA, O. (1972): Mapa Geológico de España escala l:200.000. Hoja no 33 Lérida. 31 pp. I.G.M.l. Madrid.

SANCIIO, C. (1988): Geomorfología de la Cuenca Baja del rio Cinca. Tesis Doctoral Facultad de Ciencias. Univ. de Zaragoza. 743 p. (inéd.)

SOI.Li, L. (1953 a): "Terrazas cuaternarias deformadas de la cuenca del Ebro". Mem. R. Acad. Ciencias y Artes de Barcelona, XXX, $n^{\circ} 7$, pp. 239-259. Barcelona.

SOLE., L. (1953 b): "El piedemonte de Lérida y las terrazas deformadas de sus rios". lbérica, 18, pp. 425-428 y 439. Barcelona.

SOL.E, L. y FONT, J.M. (1929): "Las terrazas del Segre en las inmediaciones de Lérida". Publ. Ins. Geol. y Topogr. Diputación Prov. Barcelona, 11, 7 p. Barcelona.

SOLE, L. (1946): "Problemas morfológicos del Llano de Lérida". Llerda, nº VI, pp. 7-22. Lérida.

SUNYER, E. (1973): "Primeras precisiones sobre la industria lítica del Segre". Geol. Med. Occid, 8 p. Barcelona

SUNYER, E. (1974): "Descubrimiento de una 'pebble culture' en cl valle del Segre" Ibérica, 141, pp. $122-124$. 